\title{
Practical guidance on the use of laboratory testing in the management of bleeding in patients receiving direct oral anticoagulants
}

This article was published in the following Dove Press journal:

Vascular Health and Risk Management

\author{
Hugo ten Cate' \\ Yvonne MC Henskens ${ }^{2}$ \\ Marcus D Lancé ${ }^{3}$ \\ 'Department of Internal Medicine, \\ Cardiovascular Research Institute, \\ ${ }^{2}$ Department of Clinical Chemistry, \\ ${ }^{3}$ Department of Anaesthesiology, \\ Maastricht University Medical Centre, \\ Maastricht, the Netherlands
}

Correspondence: Hugo ten Cate Department of Internal Medicine, Cardiovascular Research Institute, Maastricht University Medical Centre, 6200 MD, Maastricht, the Netherlands Tel +3l 433884262

Email h.tencate@maastrichtuniversity.nl

\begin{abstract}
Direct oral anticoagulants (DOACs) have demonstrated a favorable benefit-risk profile in several thromboembolic disorders and are increasingly used in routine clinical practice. A number of real-world studies on DOACs are ongoing, and data published so far have shown broadly similar outcomes to those demonstrated in the respective phase III trials. Despite their beneficial attributes, bleeding risk (as with any other anticoagulants) is often a concern for physicians when prescribing DOACs, particularly in elderly patients, those with significant comorbidities, and other high-risk patient populations. Although the absence of routine coagulation monitoring is an advantage of the DOACs, measuring their anticoagulant effect and/or plasma drug levels may be helpful in certain clinical scenarios to help patient management and improve outcomes. In this paper, practical guidance and recommendations are provided for clinical situations in which the test results may aid clinical decision-making, including patients with life-threatening bleeding events, patients without bleeding but with test results indicating a risk of bleeding, for those patients with a suspected thromboembolism while receiving a DOAC, or prior to patients undergoing elective or urgent surgical procedures. Finally, appropriate monitoring of the DOACs could be of substantial benefit to patients, and there is a high potential for development in this area in the future.
\end{abstract}

Keywords: bleeding, direct oral anticoagulants, laboratory testing, perioperative management, practical guidance

\section{Introduction}

Direct oral anticoagulants (DOACs) are increasingly used in routine clinical practice for the management of several thromboembolic disorders, including the prevention of stroke and systemic embolism in patients with nonvalvular atrial fibrillation (NVAF), and the treatment and secondary prevention of venous thromboembolism (VTE). Unlike vitamin K antagonists (VKAs), the DOACs (ie, apixaban, dabigatran, edoxaban, and rivaroxaban) have a fast onset of action and are given as fixed dosing regimens without routine coagulation monitoring because of their more predictable pharmacokinetics and pharmacodynamics. Reduced doses of the DOACs are only recommended for specific patient populations (such as those with moderate/severe renal impairment in patients with NVAF). ${ }^{1-4}$ Although the absence of routine coagulation monitoring is an advantage of the DOACs, measuring their anticoagulant effects and/or plasma drug levels may be helpful in certain clinical scenarios such as in patients with lifethreatening bleeding events, patients at a risk of bleeding, patients with suspected thromboembolism, patients about to undergo elective or urgent surgery, patients due to 
undergo thrombolysis therapy, or patients with suspected drug accumulation. ${ }^{5}$ The results of the laboratory tests could be used to aid clinical decision-making in such circumstances.

As a class, the DOACs have demonstrated an improved benefit-risk profile compared with standard-of-care warfarin for long-term anticoagulant therapy (eg, for stroke prevention in patients with NVAF) in randomized phase III clinical trials, with a significant reduction in the incidence of spontaneous intracranial hemorrhage $(\mathrm{ICH})$ and fatal bleeding. ${ }^{6-10}$ In addition, the outcomes of major bleeding events were no worse or better than in patients treated with warfarin. ${ }^{11-14}$ Currently, a number of real-world studies on DOACs are ongoing, and data published so far have shown broadly similar outcomes to those demonstrated in the phase III trials. However, despite their beneficial attributes, monitoring bleeding risk is often a concern for physicians when prescribing the DOACs, particularly in older patients (eg, $>80$ years old), those with comorbidities, and those taking other medications that may increase the risk of bleeding. There is a perception that the management of bleeding events may be more difficult in patients taking a DOAC than in those taking a VKA; this is further complicated by the lack of rapid quantitative tests to measure plasma levels of the DOACs, ${ }^{15}$ in contrast to the readily available, routinely used international normalized ratio (INR) for the VKAs. ${ }^{16}$ Even with no ongoing hemorrhage, patients receiving the DOACs may require urgent surgery or invasive procedures, which poses an additional risk of bleeding. Irrespective of the type of anticoagulant therapy (ie, oral or parenteral), rapid assessment of bleeding risk and minimizing any modifiable risk factors remain the most important patient management strategies. With four DOACs available for clinical use, selecting the right drug and right dose for each individual patient and strict medication adherence are essential for optimal clinical outcomes.

As the use of DOACs becomes more prevalent in routine clinical practice, it is even more important for physicians to understand laboratory testing and bleeding management in patients receiving these drugs. This article discusses laboratory tests for the DOACs, and how the test results can aid clinical decision-making in emergency and other specific clinical situations and provides practical guidance on optimal bleeding management.

\section{Laboratory testing of DOACs}

Standard coagulation assays such as the prothrombin time (PT) and activated partial thromboplastin time (aPTT) assays are readily available in all hospitals and can be used as first- line tests to provide a qualitative assessment of rivaroxaban and dabigatran, respectively. More recently, global coagulation assays such as viscoelastic tests (ROTEM ${ }^{\circledR}$ and TEG $^{\circledR}$ ) and thrombin generation assays have also been suggested as potential tests for the assessment of the anticoagulant effect of DOACs. ${ }^{17,18}$ These assays deliver global information on clot development, stabilization, and dissolution that signify in vivo hemostasis and have several advantages such as short turnaround time and the possibility of more accurate measurements. Quantitative assays can accurately determine plasma drug concentrations and can be used as second-line tests (if the results are not required within $24 \mathrm{~h}$ ). The measured trough plasma levels after therapeutic doses may be helpful when assessing bleeding risk (Table 1); however, these assays are currently not widely available.

\section{Rivaroxaban}

Rivaroxaban prolongs PT in a concentration-dependent manner, but the effect varies markedly with different thromboplastins because of their differing sensitivities to rivaroxaban. ${ }^{1,19,20}$ One study showed that the rank order of reagents for PT prolongation was Triniclot ${ }^{\mathrm{TM}}$ PT Excel S $>$ RecombiPlasTin $2 \mathrm{G}$ $>$ Neoplastin $\mathrm{R}^{\circledR}>$ Neoplastin CI Plus ${ }^{\circledR}>$ Triniclot $^{\mathrm{TM}}$ PT Excel $>$ Triniclot $^{\mathrm{TM}}$ PT HTF $>$ Innovin ${ }^{\circledR}$ in plasma samples spiked with rivaroxaban (concentration range $=11-1,090 \mathrm{ng} / \mathrm{mL}$ ). ${ }^{21}$ In plasma samples from patients receiving rivaroxaban for the prevention of VTE after hip or knee replacement surgery, Neoplastin CI Plus ${ }^{\circledR}$ and RecombiPlasTin 2G showed high sensitivity to the effect of rivaroxaban, whereas PT measured by using Thrombore ${ }^{\circledR} \mathrm{S}$ and Innovin ${ }^{\circledR}$ had low sensitivity to the effect of rivaroxaban. ${ }^{22}$ The general consensus is that PT (particularly with a sensitive reagent) can be used as a screening test for urgent assessment of rivaroxaban exposure (Figure 1). In this regard, Neoplastin CI Plus ${ }^{\circledR}$ is recommended for PT measurement by the European Heart Rhythm Association (EHRA) and in the rivaroxaban - Summary of Product Characteristics. ${ }^{1,5}$ Rivaroxaban also prolongs the aPTT, but this assay is less sensitive than the PT and is not generally suitable for use in patients receiving rivaroxaban. Rivaroxaban prolongs the clotting time of EXTEM (a test for the extrinsic coagulation pathway) and INTEM (a test for the intrinsic coagulation pathway) in the ROTEM ${ }^{\circledR}$ analysis in a dose-dependent fashion (which could be used to detect higher concentrations of the drug). However, there may be situations in which the tests fail to determine the therapeutic levels of rivaroxaban. ${ }^{18,23-25}$ The thrombin generation assay (TGA) also detects the effects of rivaroxaban in a dosedependent manner. ${ }^{26}$ Both tests have proven to be useful in 
Table I Pharmacokinetic characteristics of direct oral anticoagulants, methods, and indications for laboratory testing

\begin{tabular}{|c|c|c|c|c|}
\hline Characteristic & Rivaroxaban & Apixaban & Edoxaban & Dabigatran \\
\hline$T_{\max }(\mathrm{h})$ & $2-4$ & $3-4$ & $\mathrm{I}-2$ & $0.5-2$ \\
\hline Half-life (h) & $5-13$ & $\sim 12$ & $10-14$ & $12-14$ \\
\hline Renal clearance (as active drug; \%) & $\sim 33$ & $\sim 27$ & $\sim 50$ & $\sim 80$ \\
\hline \multicolumn{5}{|c|}{ Laboratory testing } \\
\hline Qualitative assessment & $\begin{array}{l}\text { PT (also affects aPTT, } \\
\text { but not suitable for use); } \\
\text { ROTEM }^{\circledR}, \text { TGA }\end{array}$ & $\begin{array}{l}\text { PT and aPTT show low sensitivity } \\
\text { in general and not suitable for } \\
\text { use; ROTEM }{ }^{\circledR} \text { or TGA }\end{array}$ & $\begin{array}{l}\text { PT and aPTT show low } \\
\text { sensitivity in general and } \\
\text { not suitable for use }\end{array}$ & $\begin{array}{l}\text { aPTT; TT (also affects PT, } \\
\text { but not suitable for use); } \\
\text { ROTEM }^{\circledR} / \text { TEG }^{\circledR}, \text { TGA }\end{array}$ \\
\hline Quantitative assessment & $\begin{array}{l}\text { Anti-factor Xa assay with } \\
\text { rivaroxaban calibrators }\end{array}$ & $\begin{array}{l}\text { Anti-factor } \text { Xa assay with } \\
\text { apixaban calibrators }\end{array}$ & $\begin{array}{l}\text { Anti-factor } X \text { a assay with } \\
\text { edoxaban calibrators }\end{array}$ & $\mathrm{dTT}$ or $\mathrm{ECT} / \mathrm{ECA}$ \\
\hline $\begin{array}{l}\text { Main indications for laboratory } \\
\text { testing }\end{array}$ & $\begin{array}{l}\text { - Prior to urgent surgeries } \\
\text { - Acute and chronic kidne } \\
\text { - Acute and chronic liver } \\
\text { - Suspected overdose or } \\
\text { - Onset of life-threatening } \\
\text { - Assessing hemostatic sta }\end{array}$ & $\begin{array}{l}\text { ey /invasive procedures } \\
\text { ey failure } \\
\text { failure } \\
\text { drug accumulation } \\
\text { g bleeding or thrombotic events } \\
\text { atus after giving antidotes }\end{array}$ & & \\
\hline
\end{tabular}

Note: Data from previous studies. ${ }^{1-4,17,18,24,27,28}$

Abbreviations: aPTT, activated partial thromboplastin time; dTT, diluted thrombin time; ECA, ecarin chromogenic assay; ECT, ecarin clotting time; PT, prothrombin time; $T_{\text {max }}$, time to maximum plasma concentration; TGA, thrombin generation assay; TT, thrombin time.

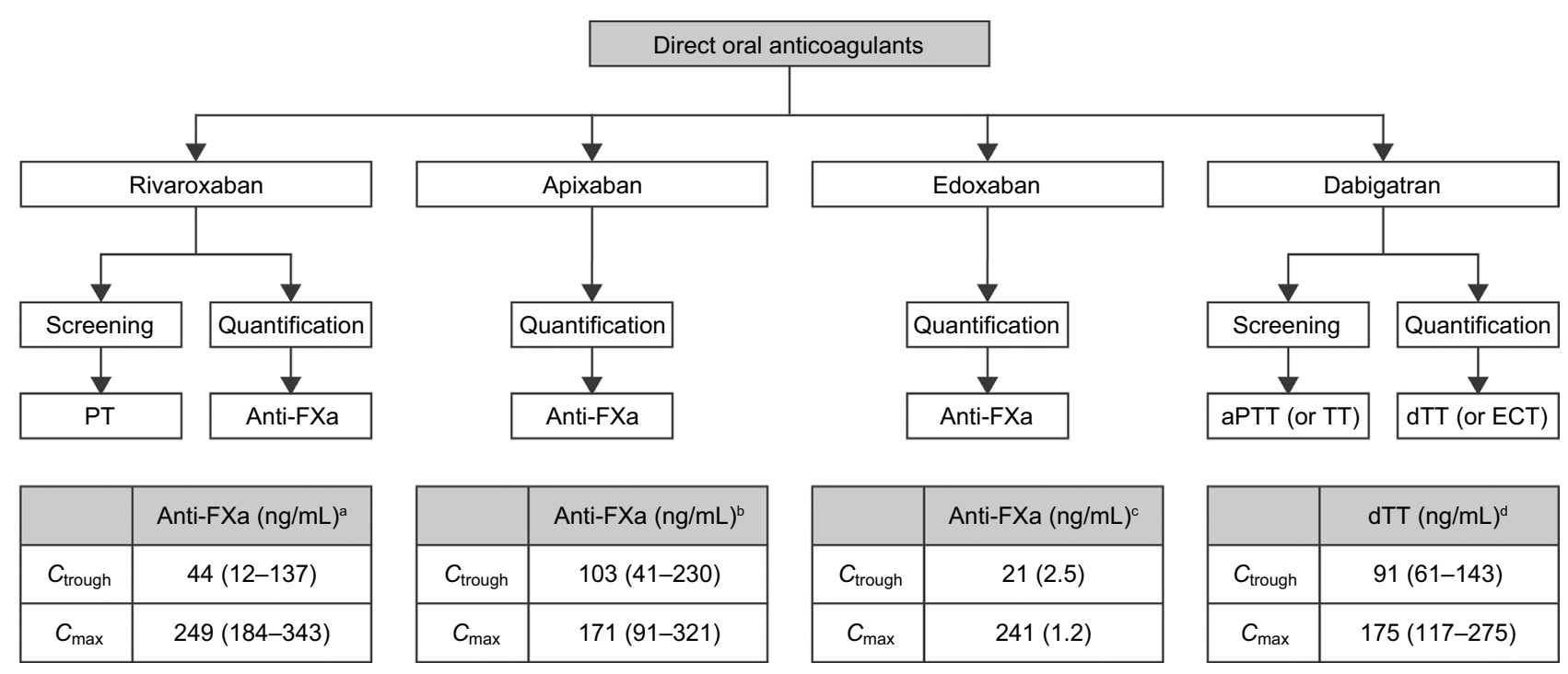

Figure I Laboratory testing of DOACs and expected plasma levels (based on published mass spectrometry data or from the Summary of Product Characteristics) after therapeutic doses.

Notes: ${ }^{\mathrm{E} E s t i m a t e d}$ geometric mean (5/95th percentile) after the administration of rivaroxaban $20 \mathrm{mg}$ once daily for stroke prevention. ${ }^{57}$ bEstimated median (5/95th percentile) after the administration of apixaban $5 \mathrm{mg}$ twice daily for stroke prevention. ${ }^{3}$ 'Estimated geometric mean (standard deviation) after the administration of edoxaban $60 \mathrm{mg}$ once daily for stroke prevention. ${ }^{58}$ Estimated geometric mean (25/75th percentile) after the administration of dabigatran I50 mg twice daily for stroke prevention. ${ }^{2}$

Abbreviations: anti-FXa, anti-factor Xa; aPTT, activated partial thromboplastin time; $C_{\text {max }}$, maximum plasma drug concentration; $C_{\text {trough }}$, trough plasma drug concentration; DOAC, direct oral anticoagulant; $\mathrm{dTT}$, diluted thrombin time; ECT, ecarin clotting time; PT, prothrombin time; TT, thrombin time.

cases of treatment with prothrombin complex concentrate (PCC).$^{29}$ However, the reports on $\mathrm{TEG}^{\circledR}$ parameters to detect rivaroxaban are inconsistent. ${ }^{17,30}$

A large number of studies have consistently demonstrated the accuracy and sensitivity of drug-specific anti-factor Xa chromogenic assays for the quantitative measurement of rivaroxaban, as summarized in a comprehensive literature review. ${ }^{31}$ These assays (calibrated with rivaroxaban) can measure a wide range of rivaroxaban plasma concentrations that cover the expected levels after therapeutic doses (Figure 1) and are, therefore, recommended by the EHRA and the rivaroxaban - Summary of Product Characteristics. ${ }^{1,5}$ However, the longer turnaround time to obtain the test results makes them less convenient than PT in emergency situations.

\section{Apixaban}

There is less published information on the laboratory testing of apixaban, compared with rivaroxaban. ${ }^{31}$ In general, routine coagulation tests show lower sensitivity to the effect 
of apixaban - most PT and aPTT reagents show only mild or modest sensitivity. As with rivaroxaban, variability in reagent sensitivity also exists. ${ }^{32,33}$ Depending on the reagent used, PT may remain normal at therapeutic concentrations of apixaban. ${ }^{34}$ Therefore, PT or aPTT assays are not recommended for estimating the relative anticoagulation intensity or plasma concentration of apixaban after therapeutic doses. The general recommendation for the assessment of apixaban exposure is anti-factor Xa chromogenic assays using specific apixaban standard calibrators (Figure 1). ${ }^{3,5}$ Recent data suggested that the ROTEM ${ }^{\circledR}$ assay and TGA may be useful for screening, with ROTEM ${ }^{\circledR}$ providing quick results in emergency situations. ${ }^{24}$ Currently, there is no suitable standard assay to be used as an initial quick screening test for apixaban in emergency situations, although the dilute Russell's viper venom time (dRVVT) has been proposed as a potential option (as well as for the other DOACs). This lack of a robust quick initial screening test for apixaban could be problematic in situations such as in patients experiencing major bleeding or prior to emergency surgery, where getting accurate information on coagulation status is important.

\section{Edoxaban}

Published data on laboratory measurement of edoxaban are limited. Similar to apixaban, edoxaban prolongs the PT and aPTT, but the changes after the expected therapeutic doses are rather small and subject to a high degree of variability. Therefore, these clotting assays are unsuitable for assessing the anticoagulant effect of edoxaban. ${ }^{4,5,35}$ Data from a recent study using spiked plasma samples (concentration range $=0-1,000 \mathrm{ng} / \mathrm{mL}$ ) suggested that PT could be informative, but the effect depended on the assay reagent. ${ }^{36}$ Another recent publication showed that PT reagents - especially Triniclot ${ }^{\mathrm{TM}}$ PT Excel $\mathrm{S}$ - had a positive correlation with edoxaban concentrations. ${ }^{37}$ The general consensus is that anti-factor Xa assays (with edoxaban calibrators) are best suited for assessing edoxaban exposure, ${ }^{4,5}$ and these assays show a linear relationship with edoxaban plasma concentrations.

\section{Dabigatran}

Dabigatran prolongs clotting assays such as aPTT and PT. The concentration-response curve for prolongation of the aPTT is curvilinear and flattens at higher concentrations $(\geq 200 \mathrm{ng} / \mathrm{mL}) .{ }^{38}$ In addition, the test results at given dabigatran concentrations vary between reagents because of their different sensitivities to dabigatran. ${ }^{39}$ Despite these limitations, the aPTT can be a useful screening assay, but because of its limited sensitivity, it is not suitable for quantification of the anticoagulant effect of dabigatran, especially at high concentrations. $^{2}$ The standard thrombin time (TT) assay is highly sensitive to the presence of dabigatran, and a normal TT value excludes the presence of even low levels of dabigatran. ${ }^{5}$ For accurate quantitative measurement of dabigatran concentrations, diluted thrombin time (dTT) tests (such as Hemoclot $^{\circledR}$, Technoview ${ }^{\circledR}$, or Hemosil ${ }^{\circledR}$ ) using dabigatran calibrators are recommended. The ecarin clotting time (ECT) assay provides a direct measure of dabigatran activity, but it is not routinely available. If the assessment of low dabigatran plasma concentrations is required (eg, in the perioperative setting), the ecarin chromogenic assay (ECA), as well as dTT and ECT assays, can be used. For general and quick screening, the viscoelastic tests ( such as ROTEM $^{\circledR}$ and TEG ${ }^{\circledR}$ ) seem to be useful and may guide treatment with PCC. ${ }^{24,40,41}$ However, TGAs are more accurate in monitoring treatment with idarucizumab. ${ }^{40}$

\section{Practical guidance on laboratory assessment of DOACs}

DOACs have relatively short half-lives compared with VKAs, and their maximum effects on clotting tests occur around their maximum plasma concentrations, at $0.5-4 \mathrm{~h}$ after tablet intake (depending on the drug; Table 1). ${ }^{1-4}$ Therefore, it is important to know when the last dose of the DOAC was administered relative to the time of blood sampling and to take this into account, as well as the clinical profile of the patient (such as renal and hepatic function). The PT and APTT assays are readily available throughout the day with a fast turnaround time for the test results; therefore, these are the currently recommended screening tests (Figure 1). However, because of the large variations in the sensitivity of the assay reagents, each laboratory should standardize and optimize their local assays. If sensitive reagents are used, normal test results may infer acceptable levels of rivaroxaban (with PT) or dabigatran (with aPTT). Neither the PT nor the aPTT assay is suitable for the measurement of the anticoagulation effects of apixaban. ${ }^{3}$ For edoxaban, the PT has been shown to be unsuitable because of high variability; however, the aPTT may be suitable for measuring the anticoagulation effects of edoxaban. ${ }^{42}$ The assessment of coagulation properties by using ROTEM $^{\circledR}$ and TEG ${ }^{\circledR}$ could provide information on the presence of the DOACs, and these assays have quick turnaround times and could be used to guide the treatment (especially in emergency situations). Although TGAs are sensitive to most of the DOACs, they are not currently standardized and are labor-intensive to use in daily practice. INR was specifically developed for monitoring VKA therapy, and therefore, point-of-care PT/INR 
testing $\left(\right.$ CoaguChek $\left.^{\circledR}\right)$ is not recommended for assessing the anticoagulation intensity of DOACs.

Some patients treated with VKAs may be transitioned to a DOAC. In such situations, the clinician may decide to measure INR prior to stopping VKA administration in order to ascertain when to initiate DOAC therapy and avoid periods of inadequate anticoagulation. ${ }^{43}$ INR values will be elevated for the first few days after transitioning because of the influence of DOACs on PT/INR and the residual effects of the VKA. However, this does not reflect the anticoagulation intensity of the DOAC, and INR testing should be stopped after the initiation of DOAC therapy.,44

Accurate estimation of exposure levels can be accomplished for dabigatran with the dTT or ECT, and ECA tests and for rivaroxaban, apixaban, and edoxaban with anti-factor Xa assays. ${ }^{5,31}$ According to the drug information in the rivaroxaban - Summary of Product Characteristics, the thresholds at trough concentration that may be associated with an increased risk of bleeding were a plasma level of $>200 \mathrm{ng} / \mathrm{mL}$ (eg, measured using the dTT test), ECT $>3.0$-fold, or aPTT $>2.0$-fold of the upper limits of the normal values in patients receiving dabigatran for stroke prevention and for the treatment of VTE. ${ }^{2}$ An analysis of plasma samples from 9,183 patients with NVAF enrolled in the RE-LY study showed an inverse correlation between ischemic events and dabigatran trough plasma concentrations. ${ }^{45}$ Previously, it has been suggested that rivaroxaban plasma levels of $>200 \mathrm{ng} / \mathrm{mL}$ measured at $\geq 12 \mathrm{~h}$ after tablet intake may indicate potential drug accumulation (eg, in patients with worsening renal function). ${ }^{22}$ There are currently no published studies on the cutoff levels for apixaban and edoxaban. A subanalysis of the ENGAGE AF-TIMI 48 trial showed that the probability of major bleeding events increased with increasing trough edoxaban concentrations of up to $150 \mathrm{ng} / \mathrm{mL} .{ }^{46}$ In general, the threshold of plasma drug level indicative of an elevated bleeding risk remains to be further established and supported by clinical evidence, and the risk factors of patients should always be considered when making clinical decisions.

dRVVT assays are available (although not at all times) in many coagulation laboratories and are used for lupus anticoagulant testing. Recent data suggested that the dRVVT might be useful in urgent situations for screening patients who are receiving a DOAC. ${ }^{47}$ It was shown that the dRVVT (using STA ${ }^{\circledR}$-Staclot ${ }^{\circledR}$ DRVV-Confirm) could provide a rapid estimation of the intensity of anticoagulation with rivaroxaban and dabigatran without any specific calibrators and can identify supratherapeutic plasma levels of these drugs. ${ }^{47}$ The potential role of the dRVVT (using a "Confirm" lupus anticoagulant reagent) for urgent testing of the DOACs has also been proposed by other studies, ${ }^{31}$ but further clinical data are required before this assay can be recommended for routine use as a first-line test for the DOACs.

According to the DOAC drug labels, neither the dose nor dosing intervals should be changed in response to changes in laboratory coagulation parameters alone for the current registered indications. Instead, a change in the type of DOAC, or a switch to a VKA, could be considered in the event that laboratory parameters suggest consistent overdosing or underdosing of a specific drug. Moreover, the metabolism and elimination of DOACs is complex, involving multiple liver and renal clearance pathways. Thus, impairment of renal and hepatic function influences DOAC exposure levels, as well as certain drug interactions (such as drugs affecting cytochrome P450 3A4 [CYP3A4] and P-glycoprotein [P-gp]). ${ }^{1-4}$ These factors should be considered when interpreting test results, and clinicians should assess whether a reduced dose is indicated (ie, in patient groups as specified in the approved labels). ${ }^{1-4}$

A number of studies have also shown the potential influence of DOACs on coagulation function assessments (eg, thrombophilia testing), and this topic has been discussed in other dedicated, comprehensive reviews, which also provide practical guidance on how to avoid diagnostic errors in patients receiving DOACs. ${ }^{31,48}$

\section{Emergency situations/ life-threatening bleeding while receiving a DOAC}

The DOACs have shown noninferiority to conventional standard therapy in phase III trials for the management of several thromboembolic indications, with a similar or better safety profile. ${ }^{49}$ For example, in patients with NVAF, the DOACs were associated with a lower risk of spontaneous ICH and other life-threatening bleeding events. ${ }^{6-10}$ Such serious adverse events, although rare, do occur in patients receiving anticoagulant therapy. In emergency situations, such as when patients treated with DOACs experience a life-threatening bleeding event, the PT (for rivaroxaban) and the aPTT or TT (for dabigatran) can be used as first-line tests to assess the levels of the respective drug in plasma. A normal PT or aPTT result with a sensitive reagent indicates that it is unlikely that the bleeding event is enhanced by rivaroxaban and dabigatran, respectively. Because of its high sensitivity, a normal TT result in dabigatran-treated patients with serious bleeding excludes the presence of clinically relevant levels of dabigatran. Depending on the urgency of 
the situation, quantitative tests can be performed to determine the drug levels in plasma (eg, using dTT or ECT/ECA for dabigatran and anti-factor Xa assays for rivaroxaban, apixaban, and edoxaban). The test results may help in clinical decision-making on whether reversal agents should be administered (in addition to other factors to be considered before making such decisions). However, it should be noted that the turnaround time of many monitoring assays may preclude their use in an emergency situation where time is critical. ${ }^{50}$ The Subcommittee on Control of Anticoagulation of the International Society on Thrombosis and Haemostasis recommended that in patients with serious bleeding, a drug concentration $>50 \mathrm{ng} / \mathrm{mL}$ is likely to be sufficiently high to warrant administration of an antidote. In patients requiring an urgent surgical intervention that is associated with a high risk of bleeding, antidote administration should be considered if the drug concentration exceeds $30 \mathrm{ng} / \mathrm{mL} .{ }^{50}$ However, it should be noted that these are expert opinions, and the cutoff levels are estimates (ie, not based on data from randomized clinical trials). Moreover, the clinical significance of an elevated measurement remains unclear; therefore, it is suggested that clinicians should interpret DOAC laboratory results with caution. ${ }^{27}$

Specific antidotes for the DOACs are in various stages of development. Idarucizumab, an antidote for dabigatran, is now approved by both the US Food and Drug Administration (FDA) and the European Medicines Agency following the phase III trial. ${ }^{51}$ Idarucizumab is indicated in adult patients treated with dabigatran when rapid reversal of its anticoagulant effects is required (ie, emergency surgery/urgent procedures and in life-threatening or uncontrolled bleeding). The recommended idarucizumab dose is $5 \mathrm{~g}$; administration of a second $5 \mathrm{~g}$ dose may be considered in situations such as recurrence of clinically relevant bleeding together with prolonged clotting times, if potential rebleeding would be life-threatening and prolonged clotting times are observed, or in patients who require a second emergency surgery/urgent procedure and have prolonged clotting times (ie, aPTT, TT, dTT, or ECT). ${ }^{2}$ No licensed antidote is currently available for the other DOACs, although andexanet alfa, an antidote for the factor Xa inhibitors, is currently under review by the FDA and has shown promise in ex vivo, animal, and healthy volunteer studies to dose dependently reverse factor $\mathrm{Xa}$ inhibition and restore thrombin generation. ${ }^{52}$ Andexanet alfa is undergoing phase III clinical investigations; the ANNEXA-4 study (NCT02329327) is evaluating the efficacy and safety of andexanet alfa for the management of serious bleeding in patients treated with rivaroxaban, apixaban, and eventually edoxaban. In addition, ciraparantag (PER977), a small molecule designed to bind unfractionated heparin, low-molecular-weight heparin, and fondaparinux, as well as the DOACs, has been reported to reverse the anticoagulant effects of all of the DOACs and is currently at an early stage of clinical development. ${ }^{50}$ It is important to note that although such reversal agents diminish DOAC activity, they have no effect on the underlying cause of bleeding.

Figure 2 outlines a general bleeding management protocol, similar to that recommended in the current AF management guidelines, including routine measures such as stopping further drug administration, local compression (if applicable), and surgical intervention. ${ }^{5}$ In cases of a severe/life-threatening bleeding event, specific antidotes (eg, idarucizumab, $5 \mathrm{~g}$, in patients treated with dabigatran) or nonspecific reversal agents, such as PCC (50 IU/kg followed by $25 \mathrm{IU} / \mathrm{kg}$, if required) or activated prothrombin complex concentrate (aPCC; $50 \mathrm{IU} / \mathrm{kg}$ ), may be considered (Figure 2). However, published studies on the nonspecific reversal agents in the reversal of DOACs have been limited to animal models and small numbers of healthy subjects. The outcomes measured after PCC and aPCC administration have largely been centered on PT, aPTT, TT, anti-factor Xa activity, and thrombin generation parameters (ie, partial or complete reversal). Although the restoration of coagulation does not necessarily translate into a good clinical outcome, these tests are helpful to assess the extent of reversal. For example, if the PT (for rivaroxaban) or aPTT/TT (for dabigatran) is still significantly prolonged after the first dose of a reversal agent (eg, a suspected "rebound" with dabigatran) ${ }^{2}$ or the plasma drug concentration is still $>50 \mathrm{ng} / \mathrm{mL},{ }^{50}$ the subsequent administration of reversal agents may be considered, particularly when there is a high risk of recurrent serious bleeding.

\section{Patients without bleeding, but test results indicate a risk of bleeding}

When the results from the screening tests in an emergency situation suggest a risk of bleeding in patients without bleeding complications (eg, marked prolongations of the PT and aPTT/TT for rivaroxaban and dabigatran, respectively), it would be helpful to perform quantitative tests to confirm the extent of drug exposure by measuring the plasma drug concentrations with anti-factor Xa assays (ie, rivaroxaban, apixaban, and edoxaban) or dTT or ECT/ECA (dabigatran). The test results should be compared with the expected plasma concentration range after the administration of therapeutic doses, although it should be noted that the ranges can vary widely and no "safe" therapeutic plasma concentrations 


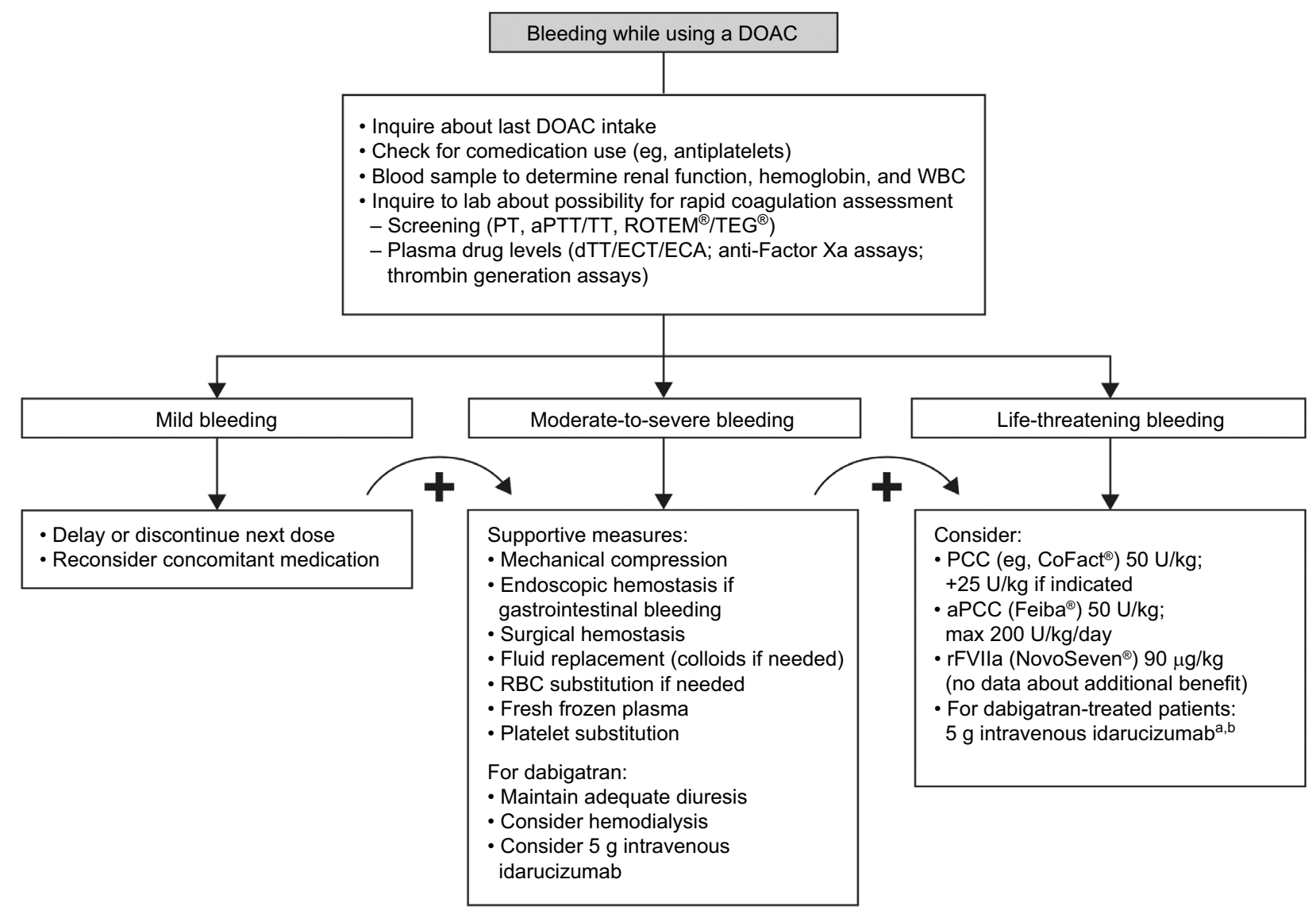

Figure 2 Protocol for the management of bleeding complications in patients receiving DOACs.

Notes: Heidbuchel H, Verhamme P, Alings M, et al. Updated European Heart Rhythm Association Practical Guide on the use of non-vitamin K antagonist anticoagulants in patients with non-valvular atrial fibrillation. Europace. 2015;17:1467-1507, ${ }^{5}$ doi: 10.1093/europace/euv309. Reproduced with permission of Oxford University Press on behalf of the European Society of Cardiology (C) The Author 2016 - All rights reserved. For permissions please email: journals.permissions@oup.com. Please visit: www. escardio.org. European Society of Cardiology was not involved in the development of this EHRA Practical Guide and in no way influenced its content. No advertisements/ pharmaceutical branding to be used in association with the material. Inclusion under a Creative Commons license allowing onward reuse is prohibited vl.4. aln a subset of patients, recurrence of plasma concentrations of unbound dabigatran and concomitant prolongation of clotting tests have occurred up to $24 \mathrm{~h}$ after the administration of idarucizumab. Administration of a second $5 \mathrm{~g}$ dose of idarucizumab may be considered in the following situations: recurrence of clinically relevant bleeding together with prolonged clotting times, if potential rebleeding would be life-threatening and prolonged clotting times are observed, or if patients require a second emergency surgery/urgent procedure and have prolonged clotting times. ${ }^{2}$ The Subcommittee on Control of Anticoagulation of the International Society on Thrombosis and Haemostasis recommend that in patients with serious bleeding, a plasma drug concentration $>50 \mathrm{ng} / \mathrm{mL}$ is likely sufficiently high to warrant antidote administration. ${ }^{50}$

Abbreviations: aPCC, activated prothrombin complex concentrate; aPTT, activated partial thromboplastin time; DOAC, direct oral anticoagulant; dTT, diluted thrombin time; ECA, ecarin chromogenic assay; ECT, ecarin clotting time; PCC, prothrombin complex concentrate; PT, prothrombin time; RBC, red blood cell; rFVIla, recombinant factor VIla; TT, thrombin time; WBC, white blood cell.

have been identified. Given the relatively short half-life of the DOACs, a "wait-and-see" strategy can be advocated depending on the measured plasma drug levels. ${ }^{5}$ At the same time, as outlined before, all the factors influencing plasma activity levels, including impaired renal and liver functions, potential drug-drug interactions, and dose and timing of drug intake, should be assessed. Potential drug-drug interactions include concomitant drugs that affect the CYP3A4 and/or P-gp pathways (including certain antibiotics). Particularly in elderly patients with concurrent disease associated with a temporary deterioration in renal or hepatic function, the treating physician might consider temporary adjustments in DOAC administration, if recommended in the respective Summary of Product Characteristics. This may include a reduced dose (eg, based on their creatinine clearance $[\mathrm{CrCl}]$ levels) for the duration of the impairment. In such situations, it may be prudent to repeat clotting tests and to reevaluate the appropriateness of dose adjustment as soon as the patient (and renal function) recovers. In general, in patients without an obvious bleeding (risk), the use of prohemostatic agents or antidotes will not be indicated.

\section{Patients with a suspected thromboembolism while receiving a DOAC}

Although the risk of thromboembolic events is greatly reduced in patients who are adequately anticoagulated, events do still occur in some patients, particularly if the patient is receiving suboptimal anticoagulant treatment. If a thrombotic event occurs in patients receiving a DOAC (eg, stroke or deep 
vein thrombosis), laboratory testing is of clinical value. In this situation, quantitative tests (rather than qualitative tests) are more appropriate. If the drug level is lower than the expected value, potential drug-drug interactions should be checked, such as concomitant use of strong CYP3A4/P-gp inducers (eg, rifampicin and St. John's wort). ${ }^{5}$ It is also important to check the patient's compliance to the prescribed DOACs, including the drug administration (eg, taking with food for the 15 and $20 \mathrm{mg}$ doses of rivaroxaban), dose, and frequency (once or twice daily). Continued and dedicated patient education is a key to good adherence for optimal outcomes. If noncompliance and other potential factors are largely ruled out, a switch to another DOAC or VKA should be considered, or temporary use of LMWH should be considered. ${ }^{53}$

\section{Prior to elective and urgent surgical procedures}

Compared with the VKAs, perioperative management in patients receiving DOACs is simpler because of their relatively short half-life and more predictable pharmacokinetics and pharmacodynamics. According to the EHRA practical guidelines on the use of DOACs in patients with NVAF, ${ }^{5}$ factor Xa inhibitors should be stopped $\geq 24 \mathrm{~h}$ prior to surgery with a low bleeding risk and $\geq 48 \mathrm{~h}$ prior to surgery with a high bleeding risk. However, the abstention time should be longer ( $\geq 36$ and $\geq 48 \mathrm{~h}$, respectively) in patients with severe renal impairment $(\mathrm{CrCl}=15-30 \mathrm{~mL} / \mathrm{min})$. Because of its predominantly renal elimination, patients receiving dabigatran should allow $\geq 36$ (low risk) and $\geq 72 \mathrm{~h}$ (high risk) if their $\mathrm{CrCl}$ is $50-80 \mathrm{~mL} / \mathrm{min}$ and $\geq 48$ and $\geq 96 \mathrm{~h}$, respectively, if their $\mathrm{CrCl}$ is $30-50 \mathrm{~mL} / \mathrm{min}$. In general, laboratory testing is not necessary in most patients if these protocols are followed correctly. However, in high-risk patients (eg, those undergoing major neurological, cardiac, or vascular surgeries), the measurement of plasma drug levels prior to surgery may be helpful (Figure 3). A cutoff level ( $\leq 30 \mathrm{ng} / \mathrm{mL}$ ) for dabigatran and rivaroxaban before surgery has been proposed previously. ${ }^{54}$

The Subcommittee on Control of Anticoagulation of the International Society on Thrombosis and Haemostasis recommended the use of antidotes - if the drug concentration

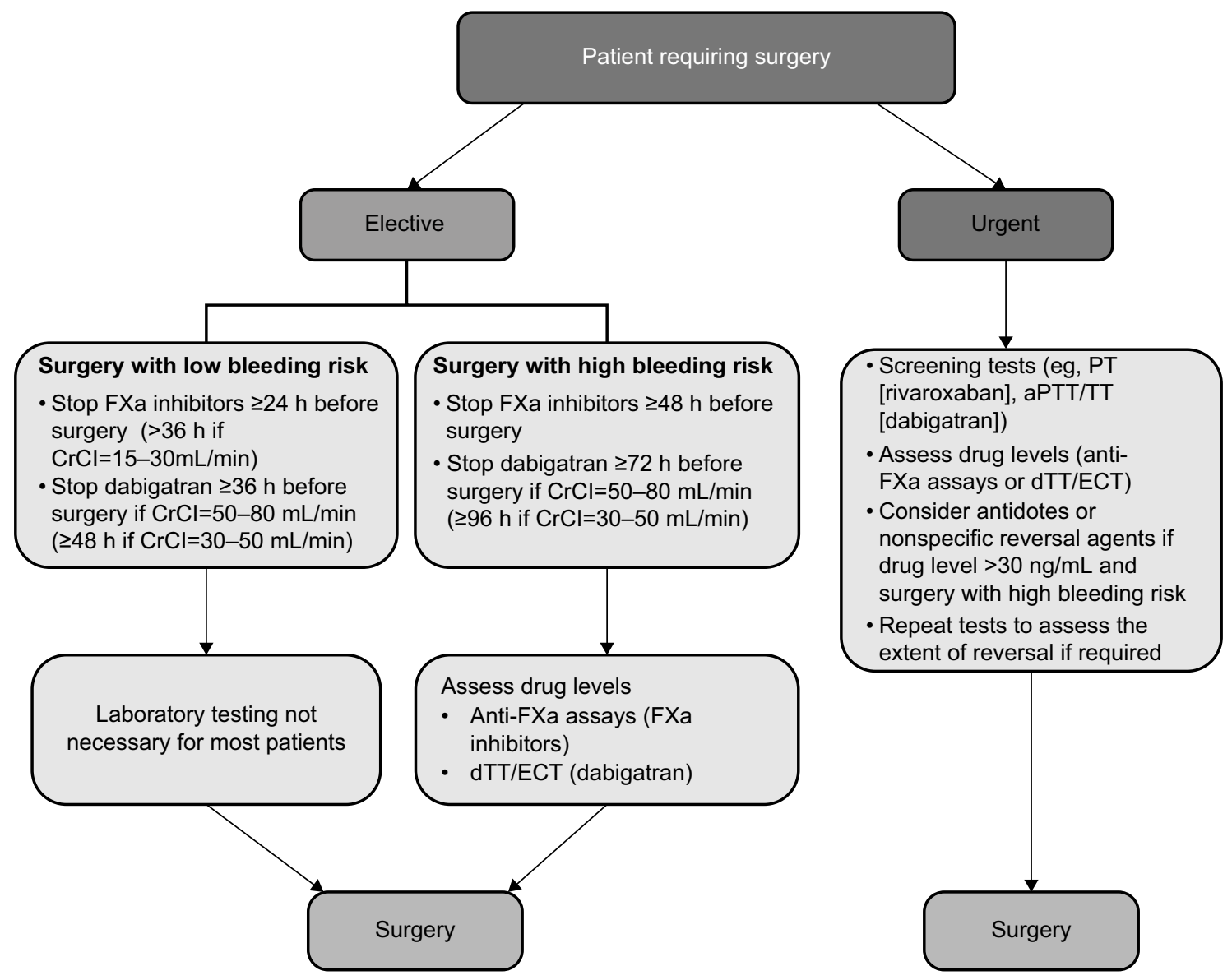

Figure 3 Patients requiring elective or urgent surgery.

Note: Data from Heidbuchel et al, ${ }^{5}$ and Hass et al. ${ }^{44}$

Abbreviations: Anti-FXa, anti-factor $\mathrm{Xa}$; aPTT, activated partial thromboplastin time; $\mathrm{CrCl}$, creatinine clearance; dTT, diluted thrombin time; ECT, ecarin clotting time; PT, prothrombin time. 
exceeds $30 \mathrm{ng} / \mathrm{mL}^{50}$ in patients who need an urgent surgical procedure that has a high risk of bleeding and cannot be delayed to allow for drug clearance. Other situations include emergency surgery or intervention in patients at a high risk of procedural bleeding, such as neurosurgery (intracranial, extradural, or spinal), lumbar puncture, cardiac, vascular (aortic dissection/aneurysm repair), hepatic, or other major organ surgery. ${ }^{50}$ In such situations, nonspecific reversal agents (eg, PCC) may be considered if specific antidotes are not available (Figure 2). Screening tests can be used in the first instance, which can be confirmed with a quantitative test if there is a need to assess the extent of the reversal. However, as noted previously, plasma drug concentrations can vary widely, calling into question the use of stringent cutoff points to influence perioperative management decisions.

\section{Suspected overdose}

It is important to distinguish between an overdose with and without bleeding complications; in the case of bleeding complications associated with an overdose, standard protocols for bleeding management should be followed. In the cases of suspected overdose without bleeding, where the anticoagulant is known but the amount ingested is unknown, quantitative assays (eg, dTT for dabigatran and anti-factor Xa assays for the direct factor Xa inhibitors) can be used to assess the exposure levels (taking into account the time of intake) and to determine how long the patient should wait before taking the next dose. For example, if the plasma level is 6-fold higher, the patient may need to wait for 5-6 days before resuming the DOAC (based on the elimination half-life). Renal function testing may also be helpful, especially in patients with known chronic renal diseases because the elimination time of the DOAC (dabigatran in particular) will be longer in these patients. The use of activated charcoal may reduce the continued absorption of DOACs within the first hours after intake. A short-term follow-up will be needed, and in some patients, the use of proton-pump inhibitors may be helpful to reduce the risk of gastrointestinal bleeding events. As a result of limited absorption, a ceiling effect with no further increase in average plasma exposure is seen at supratherapeutic doses of rivaroxaban ( $\geq 50 \mathrm{mg}$ ). ${ }^{55}$ Cases of overdosing of rivaroxaban up to $600 \mathrm{mg}$ have been reported without bleeding complications or other adverse reactions. ${ }^{1}$ No such data are currently available for the other DOACs. Hemodialysis can be used for the removal of dabigatran from plasma, but not for the direct factor Xa inhibitors.

\section{Future needs in laboratory testing of DOACs}

There remains an unmet need to develop global assay(s) for all anticoagulants. It would be helpful to have a simple, standardized assay with a fast turnaround time that could be available $24 \mathrm{~h}$ a day, which could be used to measure coagulation status and the drug levels of all oral anticoagulants in emergency situations using routinely available coagulation equipment. This would also be of clinical value after the administration of reversal agents (both specific antidotes and nonspecific reversal agents, such as PCC) to guide clinical decisions on whether to administer subsequent doses. Given the limitations of the PT and aPTT assays as universal screening tests, efforts have been made to identify better assays for this purpose. The dRVVT (using a "Confirm" lupus anticoagulant reagent) has been suggested as a potential screening test for the DOACs in emergency situations without the use of specific calibrators. ${ }^{47}$ In addition, a recent study showed the potential of a dilute Fiix-PT (dFiix-PT; using highly diluted thromboplastins, 1:1,156) for simple and rapid testing of different classes of anticoagulants, including the DOACs dabigatran, rivaroxaban, and apixaban. ${ }^{56}$ In this study, the dFiixPT was linearly sensitive to dabigatran $(32-475 \mathrm{ng} / \mathrm{mL}$ ), rivaroxaban (up to $457 \mathrm{ng} / \mathrm{mL}$ ), and apixaban (up to $572 \mathrm{ng} / \mathrm{mL}$ ). The test was then used to measure the concentrations of dabigatran, rivaroxaban, and apixaban in samples obtained from patients, and the results were compared with those obtained using dTT and anti-factor Xa assays, respectively. Linear relationships were observed when correlating the dFiix-PT with dTT (for dabigatran) and anti-factor Xa assay (for rivaroxaban); apparent linear relationships were also observed for apixaban, but the results were inconclusive because of a low number of patient samples. It was concluded that the dFiix-PT assay could provide an estimation of drug concentrations in the range of 30-200 $\mathrm{ng} / \mathrm{mL}$ for the three DOACs tested, but at higher concentrations, sample dilutions with normal plasma are needed or the results could be reported as $>200 \mathrm{ng} / \mathrm{mL}$. The authors proposed that the test could easily be automated using standard coagulation equipment and could be applied in practice as a first screening test (eg, in emergency situations) to estimate the anticoagulant levels of several DOACs (as well as of VKAs) ${ }^{56}$ However, further studies are required to confirm the clinical utility of this method and other potential screening assays. In addition, although clinical guidance on bleeding management in patients receiving the DOACs are available in the current guidelines and many consensus documents, universal, standardized protocols for bleeding management in patients receiving DOACs, including detailed 
information on the use of nonspecific reversal agents and antidotes and means of testing the effect of these agents, can further assist with patient management.

\section{Conclusion}

Although the use of DOACs does not require routine coagulation monitoring, it has become apparent that in certain clinical situations testing can help clinical decision-making and optimal patient management. There are existing assays that can be used for qualitative and quantitative assessments of the DOACs, providing an opportunity for simple, fast, and accurate universal tests for the DOACs that can be performed expeditiously in emergency settings, including assessing the extent of reversal after the administration of specific antidotes or nonspecific reversal agents. However, continued patient education remains indispensable for the success of DOAC therapy.

\section{Acknowledgments}

The authors would like to acknowledge Yong-Ling Liu for editorial support with funding from Bayer AG and Janssen Scientific Affairs, LLC. The authors had full control of, and responsibility for, the content.

\section{Disclosure}

$\mathrm{HtC}$ is a consultant to Stago and has received payments for presentations and advisory board participation from Bayer, Boehringer, Pfizer, and Daiichi Sankyo. HtC is Chairman of the Board of the Dutch Federation of Anticoagulation Clinics, which is an unpaid position, and is also a Fellow of the Gutenberg Research College, Center for Thrombosis and Hemostasis (CTH), Mainz, Germany. The other authors report no conflicts of interest in this work.

\section{References}

1. Bayer Pharma AG. Xarelto ${ }^{\circledR}$ (rivaroxaban) Summary of Product Characteristics; 2016. Available from: http://www.ema.europa.eu/docs/en_GB/ document_library/EPAR_-_Product_Information/human/000944/ WC500057108.pdf. Accessed April 13, 2017.

2. Boehringer Ingelheim International GmbH. Pradaxa ${ }^{\circledR}$ (dabigatran etexilate) Summary of Product Characteristics; 2017. Available from: http:// www.ema.europa.eu/docs/en_GB/document_library/EPAR_-_Product_Information/human/000829/WC500041059.pdf. Accessed April 13, 2017.

3. Bristol-Myers Squibb and Pfizer. Eliquis ${ }^{\circledR}$ (apixaban) Summary of Product Characteristics; 2017. Available from: http://www.ema.europa. eu/docs/en_GB/document_library/EPAR_-_Product_Information/ human/002148/WC500107728.pdf. Accessed April 13, 2017.

4. Daiichi Sankyo Europe GmbH. Lixiana ${ }^{\circledR}$ (edoxaban) Summary of Product Characteristics; 2016. Available from: http://www.ema.europa. eu/docs/en_GB/document_library/EPAR_-_Product_Information/ human/002629/WC500189045.pdf. Accessed April 13, 2017.
5. Heidbuchel H, Verhamme P, Alings M, et al. Updated European Heart Rhythm Association Practical Guide on the use of non-vitamin K antagonist anticoagulants in patients with non-valvular atrial fibrillation. Europace. 2015;17:1467-1507.

6. Connolly SJ, Ezekowitz MD, Yusuf S, et al. Dabigatran versus warfarin in patients with atrial fibrillation. N Engl J Med. 2009;361:1139-1151.

7. Giugliano RP, Ruff CT, Braunwald E, et al. Edoxaban versus warfarin in patients with atrial fibrillation. $N$ Engl J Med. 2013;369:2093-2104.

8. Granger CB, Alexander JH, McMurray JJ, et al. Apixaban versus warfarin in patients with atrial fibrillation. NEngl J Med. 2011;365:981-992.

9. Ruff CT, Giugliano RP, Braunwald E, et al. Comparison of the efficacy and safety of new oral anticoagulants with warfarin in patients with atrial fibrillation: a meta-analysis of randomised trials. Lancet. 2014;383:955-962.

10. Patel MR, Mahaffey KW, Garg J, et al. Rivaroxaban versus warfarin in nonvalvular atrial fibrillation. N Engl J Med. 2011;365:883-891.

11. Hankey GJ, Stevens SR, Piccini JP, et al. Intracranial hemorrhage among patients with atrial fibrillation anticoagulated with warfarin or rivaroxaban: the rivaroxaban once daily, oral, direct Factor Xa inhibition compared with vitamin $\mathrm{K}$ antagonism for prevention of stroke and embolism trial in atrial fibrillation. Stroke. 2014;45:1304-1312.

12. Hart RG, Diener HC, Yang S, et al. Intracranial hemorrhage in atrial fibrillation patients during anticoagulation with warfarin or dabigatran: the RE-LY trial. Stroke. 2012;43:1511-1517.

13. Hylek EM, Held C, Alexander JH, et al. Major bleeding in patients with atrial fibrillation receiving apixaban or warfarin: the ARISTOTLE trial (Apixaban for Reduction in Stroke and Other Thromboembolic Events in Atrial Fibrillation): predictors, characteristics, and clinical outcomes. J Am Coll Cardiol. 2014;63:2141-2147.

14. Piccini JP, Garg J, Patel MR, et al. Management of major bleeding events in patients treated with rivaroxaban vs. warfarin: results from the ROCKET AF trial. Eur Heart J. 2014;35:1873-1880.

15. Lippi G, Ardissino D, Quintavalla R, Cervellin G. Urgent monitoring of direct oral anticoagulants in patients with atrial fibrillation: a tentative approach based on routine laboratory tests. JThromb Thrombolysis. 2014; 38:269-274.

16. Ageno W, Gallus AS, Wittkowsky A, Crowther M, Hylek EM, Palareti G. Oral anticoagulant therapy: antithrombotic therapy and prevention of thrombosis, 9th ed: American College of Chest Physicians evidencebased clinical practice guidelines. Chest. 2012;141:e44S-e88S.

17. Dias JD, Norem K, Doorneweerd DD, Thurer RL, Popovsky MA, Omert LA. Use of thromboelastography (TEG) for detection of new oral anticoagulants. Arch Pathol Lab Med. 2015;139:665-673.

18. Chojnowski K, Gorski T, Robak M, Trelinski J. Effects of rivaroxaban therapy on ROTEM coagulation parameters in patients with venous thromboembolism. Adv Clin Exp Med. 2015;24:995-1000.

19. Samama MM, Martinoli JL, Le Flem L, et al. Assessment of laboratory assays to measure rivaroxaban - an oral, direct factor Xa inhibitor. Thromb Haemost. 2010;103:815-825.

20. Hill RA, Chung H, George E, Longson C, Stevens A. Prasugrel for the treatment of acute coronary syndromes with percutaneous coronary intervention: NICE technology appraisal guidance. Heart. 2010; 96:1407-1408.

21. Douxfils J, Mullier F, Loosen C, Chatelain C, Chatelain B, Dogné JM. Assessment of the impact of rivaroxaban on coagulation assays: laboratory recommendations for the monitoring of rivaroxaban and review of the literature. Thromb Res. 2012;130:956-966.

22. Lindhoff-Last E, Ansell J, Spiro T, Samama MM. Laboratory testing of rivaroxaban in routine clinical practice: when, how, and which assays. Ann Med. 2013;45:423-429.

23. Casutt M, Konrad C, Schuepfer G. Effect of rivaroxaban on blood coagulation using the viscoelastic coagulation test ROTEM. Anaesthesist. 2012;61:948-953.

24. Eller T, Busse J, Dittrich M, et al. Dabigatran, rivaroxaban, apixaban, argatroban and fondaparinux and their effects on coagulation POC and platelet function tests. Clin Chem Lab Med. 2014;52:835-844. 
25. Oswald E, Velik-Salchner C, Innerhofer P, et al. Results of rotational thromboelastometry, coagulation activation markers and thrombin generation assays in orthopedic patients during thromboprophylaxis with rivaroxaban and enoxaparin: a prospective cohort study. Blood Coagul Fibrinolysis. 2015;26:136-144.

26. Molenaar PJ, Dinkelaar J, Leyte A. Measuring rivaroxaban in a clinical laboratory setting, using common coagulation assays, Xa inhibition and thrombin generation. Clin Chem Lab Med. 2012;50:1799-1807.

27. Garcia D, Barrett YC, Ramacciotti E, Weitz JI. Laboratory assessment of the anticoagulant effects of the next generation of oral anticoagulants. J Thromb Haemost. 2013;11(2):245-252.

28. Salmonson T, Dogne JM, Janssen H, Burgos JG, Blake P. Non-vitaminKoral anticoagulants and laboratory testing: now and in the future. Eur Heart J Cardiovasc Pharmacother. 2017;3(1):42-47.

29. Dinkelaar J, Molenaar PJ, Ninivaggi M, de Laat B, Brinkman HJ, Leyte A. In vitro assessment, using thrombin generation, of the applicability of prothrombin complex concentrate as an antidote for Rivaroxaban. $J$ Thromb Haemost. 2013;11(6):1111-1118.

30. Rathbun S, Tafur A, Grant R, Esmon N, Mauer K, Marlar RA. Comparison of methods to determine rivaroxaban anti-Factor Xa activity. Thromb Res. 2015;135:394-397.

31. Favaloro EJ, Lippi G. Laboratory testing in the era of direct or nonvitamin $\mathrm{K}$ antagonist oral anticoagulants: a practical guide to measuring their activity and avoiding diagnostic errors. Semin Thromb Hemost. 2015;41:208-227.

32. Hillarp A, Gustafsson KM, Faxälv L, et al. Effects of the oral, direct factor Xa inhibitor apixaban on routine coagulation assays and anti-FXa assays. J Thromb Haemost. 2014;12:1545-1553.

33. Tripodi A, Padovan L, Testa S, et al. How the direct oral anticoagulant apixaban affects hemostatic parameters. Results of a multicenter multiplatform study. Clin Chem Lab Med. 2015;53:265-273.

34. Douxfils J, Chatelain C, Chatelain B, Dogné JM, Mullier F. Impact of apixaban on routine and specific coagulation assays: a practical laboratory guide. Thromb Haemost. 2013;110:283-294.

35. Cuker A, Husseinzadeh H. Laboratory measurement of the anticoagulant activity of edoxaban: a systematic review. JThromb Thrombolysis. 2015;39:288-294.

36. Douxfils J, Chatelain B, Chatelain C, Dogné JM, Mullier F. Edoxaban: impact on routine and specific coagulation assays. A practical laboratory guide. Thromb Haemost. 2016;115:368-381.

37. Iba T, Emmi M, Hiki M, et al. Comparison of prothrombin time tests used in the monitoring of edoxaban and their evaluation as indicators of the reversal effect. Int J Hematol. 2016;103:665-672.

38. van Ryn J, Stangier J, Haertter S, et al. Dabigatran etexilate - a novel, reversible, oral direct thrombin inhibitor: interpretation of coagulation assays and reversal of anticoagulant activity. Thromb Haemost. 2010;103:1116-1127.

39. Douxfils J, Mullier F, Robert S, Chatelain C, Chatelain B, Dogné JM. Impact of dabigatran on a large panel of routine or specific coagulation assays. Laboratory recommendations for monitoring of dabigatran etexilate. Thromb Haemost. 2012;107:985-997.

40. Honickel M, Treutler S, van Ryn J, Tillmann S, Rossaint R, Grottke O. Reversal of dabigatran anticoagulation ex vivo: Porcine study comparing prothrombin complex concentrates and idarucizumab. Thromb Haemost 2015;113:728-740.

41. Solbeck S, Ostrowski SR, Stensballe J, Johansson PI. Thrombelastography detects dabigatran at therapeutic concentrations in vitro to the same extent as gold-standard tests. Int J Cardiol. 2016;208:14-18.
42. Morishima Y, Kamisato C. Laboratory measurements of the oral direct factor Xa inhibitor edoxaban: comparison of prothrombin time, activated partial thromboplastin time, and thrombin generation assay. Am J Clin Pathol. 2015;143:241-247.

43. Burnett AE, Mahan CE, Vazquez SR, Oertel LB, Garcia DA, Ansell J. Guidance for the practical management of the direct oral anticoagulants (DOACs) in VTE treatment. J Thromb Thrombolysis. 2016;41: 206-232.

44. Haas S, Bode C, Norrving B, Turpie AGG. Practical guidance for using rivaroxaban in patients with atrial fibrillation: balancing benefit and risk Vasc Health Risk Manag. 2014;10:101-114.

45. Reilly PA, Lehr T, Haertter S, et al. The effect of dabigatran plasma concentrations and patient characteristics on the frequency of ischemic stroke and major bleeding in atrial fibrillation patients: the RE-LY Trial (Randomized Evaluation of Long-Term Anticoagulation Therapy). J Am Coll Cardiol. 2014;63:321-328.

46. Ruff CT, Giugliano RP, Braunwald E, et al. Association between edoxaban dose, concentration, anti-Factor Xa activity, and outcomes: an analysis of data from the randomised, double-blind ENGAGE AFTIMI 48 trial. Lancet. 2015;385:2288-2295.

47. Douxfils J, Chatelain B, Hjemdahl P, et al. Does the Russell Viper Venom time test provide a rapid estimation of the intensity of oral anticoagulation? A cohort study. Thromb Res. 2015;135:852-860.

48. Tsakiris DA. Direct oral anticoagulants - interference with laboratory tests and mechanism of action. Semin Hematol. 2014;51:98-101.

49. Weitz JI, Pollack CV Jr. Practical management of bleeding in patients receiving non-vitamin $\mathrm{K}$ antagonist oral anticoagulants. Thromb Haemost. 2015;114:1113-1126.

50. Levy JH, Ageno W, Chan NC, et al. When and how to use antidotes for the reversal of direct oral anticoagulants: guidance from the SSC of the ISTH. J Thromb Haemost. 2016;14:623-627.

51. Pollack CV Jr, Reilly PA, Eikelboom J, et al. Idarucizumab for dabigatran reversal. N Engl J Med. 2015;373:511-520.

52. Ghadimi K, Dombrowski KE, Levy JH, Welsby IJ. Andexanet alfa for the reversal of Factor Xa inhibitor related anticoagulation. Expert Rev Hematol. 2016;9:115-122.

53. Kearon C, Akl EA, Ornelas J, et al. Antithrombotic therapy for VTE disease: CHEST guideline and expert panel report. Chest. 2016;149:315-352.

54. Pernod G, Albaladejo P, Godier A, et al. Management of major bleeding complications and emergency surgery in patients on long-term treatment with direct oral anticoagulants, thrombin or Factor-Xa inhibitors: proposals of the Working Group on Perioperative Haemostasis (GIHP) - March 2013. Arch Cardiovasc Dis. 2013;106:382-393.

55. Kubitza D, Becka M, Roth A, Mueck W. Dose-escalation study of the pharmacokinetics and pharmacodynamics of rivaroxaban in healthy elderly subjects. Curr Med Res Opin. 2008;24:2757-2765.

56. Letertre LR, Gudmundsdottir BR, Francis CW, et al. A single test to assay warfarin, dabigatran, rivaroxaban, apixaban, unfractionated heparin and enoxaparin in plasma. J Thromb Haemost. 2016;14:1043-1053.

57. Mueck W, Stampfuss J, Kubitza D, Becka M. Clinical pharmacokinetic and pharmacodynamic profile of rivaroxaban. Clin Pharmacokinet. 2014;53:1-16

58. Song S, Kang D, Halim AB, Miller R. Population pharmacokineticpharmacodynamic modeling analysis of intrinsic FXa and bleeding from edoxaban treatment. J Clin Pharmacol. 2014;54:910-916.
Vascular Health and Risk Management

\section{Publish your work in this journal}

Vascular Health and Risk Management is an international, peerreviewed journal of therapeutics and risk management, focusing on concise rapid reporting of clinical studies on the processes involved in the maintenance of vascular health; the monitoring, prevention and treatment of vascular disease and its sequelae; and the involvement of
Dovepress

metabolic disorders, particularly diabetes. This journal is indexed on PubMed Central and MedLine. The manuscript management system is completely online and includes a very quick and fair peer-review system, which is all easy to use. Visit http://www.dovepress.com/ testimonials.php to read real quotes from published authors. 